\title{
Extension of the Rotating Planar Waveguide Model to Formation of Interference Pattems in Optical Fibers
}

\author{
Antonio Peña-García ${ }^{1 *}$, Francisco Pérez-Ocón ${ }^{2}$, and José Ramón Jiménez ${ }^{2}$ \\ ${ }^{1}$ Departamento de Ingeniería Civil, ETSICCP, Universidad de Granada, Granada 18071, Spain \\ ${ }^{2}$ Departamento de Óptica, Facultad de Ciencias, Universidad de Granada, Granada 18071, Spain
}

(Received February 7, 2011 : revised March 14, 2011 : accepted March 14, 2011)

\begin{abstract}
After the successful extension of the rotating planar waveguide model to Wave Optics, where a clear identification between the angular velocity of one hypothetical waveguide and the phase differences between two points on the path of one bounded mode was found, an application of the model to explain the interference theory is presented. We demonstrate that, for two bounded modes to create an interference pattern, a constrain to the relative parameters among both is imposed by the fiber optics. This result, not reported in the literature up to date, provides a new horizon to understand the interaction light-light when propagated within optical fibers.
\end{abstract}

Keywords : Fiber optics, Interference theory, Waveguides

OCIS codes : (060.2310) Fiber optics; (060.2270) Fiber characterization

\section{INTRODUCTION}

In previous works, we have developed a model that relates the propagation parameters of one given bounded ray in one optical fiber with the kinematic properties of a hypothetical planar waveguide containing the fiber $[1,2]$. This model, that provides a correspondence between each ray and one angular velocity, enables complex expressions of propagation to be determined in a simple way, in cases as sophisticated as gradient-index fibers [2].

Nevertheless, this model is not limited exclusively to Geometric Optics (that is, rays), but can also satisfactorily explain propagation of modes within the broader framework of Wave Optics. Thus, we formulated a simple expression that relates the phase difference between two different fronts of the same mode that propagates through a step-index fiber and the rotation velocity of the hypothetical waveguide bearing this optical fiber [3].

After these positive results, and given the increasing interest of models introducing rotating properties in the field of bounded radiation [4], it is worth asking whether similar relationships can be found to apply to two different modes, i.e. to relate the angular velocities corresponding to each mode with a phase difference between any two points of their paths.
This question becomes crucial in the case of coherent modes. In fact, the study of phenomena such as interference or diffraction by the powerful tool that the rotating-planarwaveguide model turns out to be, in addition to constituting an unexplored terrain, offers a new way of understanding these phenomena.

In the following sections, we show how information can be gathered regarding the phase difference between the two modes on the plane perpendicular to the fiber that may well be the one on which the interference pattern will be projected.

\section{RESULTS}

Let there be one ray (at the beginning we will consider the geometrical aspects of the problem to join Wave Optics later) emitted by a light source, which is divided into two by any of the classical methods used to achieve interferences. If, after the light is separated into two different rays $S$ and $S^{\prime}$, each with its own phase, we direct both rays into an optical fiber, we can transport these rays and afterwards use them to establish interference patterns, because they would correspond to coherent waves.

\footnotetext{
*Corresponding author: pgarcia@ugr.es

Color versions of one or more of the figures in this paper are available online.
} 


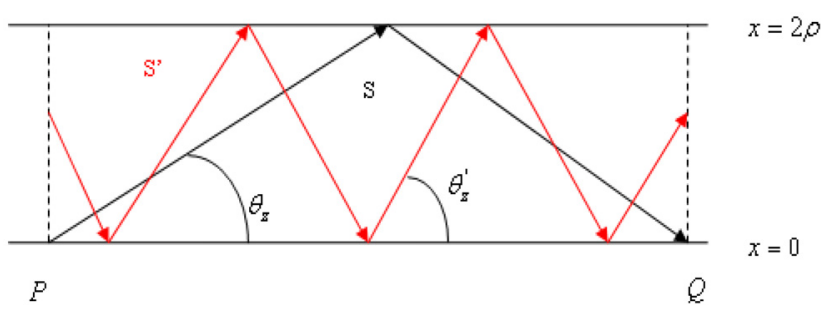

FIG. 1. Projection of two rays in different time phases in an optical fiber having a step index profile.

Let $S$ and $S^{\prime}$ be the rays resulting from the division of the wavefront of the original ray that are propagated through an optical fiber with a step-index profile with a $n_{c o}$ refraction index and radius $\rho$. Let $\theta_{z}$ and $\theta_{\phi}$ as well as $\theta^{\prime}{ }_{z}$ and $\theta^{\prime}{ }_{\phi}$ be the respective angles of propagation of both rays through this fiber as defined in [3] (see their projection in Fig. 1).

Below, we will determine the difference in optical paths between the two rays within the stretch composed of two sections of fiber determined by the points $P$ and $Q$.

As we demonstrated previously [1], the optical path followed by the rays $S$ and $S^{\prime}$ in the trajectory $\overline{P Q}$ would be:

$$
\begin{aligned}
\Delta_{S} & =\frac{\bar{P} \bar{Q}}{\cos \theta_{z} \sin \theta_{\phi}} \\
\Delta_{S^{\prime}} & =\frac{\bar{P} \bar{Q}}{\cos \theta_{z}^{\prime} \sin \theta_{\phi}^{\prime}}
\end{aligned}
$$

Therefore, the difference in optical paths between the two would be:

$$
\Delta_{S, S^{\prime}}=\bar{P} \bar{Q} n_{c o}\left(\frac{1}{\cos \theta_{z} \sin \theta_{\phi}}-\frac{1}{\cos \theta_{z}^{\prime} \sin \theta_{\phi}^{\prime}}\right)
$$

Let us consider the hypothetical rotating waveguide bearing the optical fiber under study.

Given that the fiber profile is of the step-index type, the angular velocity of this waveguide is given by [3]:

$$
\omega=\frac{c \theta_{\phi} \sin \theta_{z}}{\rho n_{c o} \sin \theta_{\phi}}
$$

Let us also conside]r the relationship between the phase difference and the optical-path difference [5], which allows us to relate rays and waves:

$$
\delta=\frac{2 \pi}{\lambda} \Delta
$$

Combining expressions (3), (4), and (5) while taking into account the relation

$$
\bar{P} \bar{Q}=\frac{4 \rho}{\operatorname{tg} \theta_{z}}
$$

we get the expression for the phase difference between any two coherent waves after following the path $\overline{P Q}$ :

$$
\delta_{S, S^{\prime}}=\frac{8 \pi n_{c o}^{2} \rho^{2}}{\lambda c}\left(\frac{\omega}{\theta_{\phi} \sin ^{2} \theta_{z}}-\frac{\omega^{\prime}}{\theta_{\phi}^{\prime} \sin ^{2} \theta_{z}^{\prime}}\right)
$$

Now, given that the interference condition implies

$$
\begin{aligned}
& o_{S, S^{\prime}}=\angle m \pi \text { for maxima } \\
& \delta_{S, S^{\prime}}=(2 m+1) \pi \text { for minima }
\end{aligned}
$$

Introducing the conditions (8a) and (8b) into expression (7), we finally get:

$$
\begin{aligned}
& \frac{\omega}{\theta_{\phi} \sin ^{2} \theta_{z}}-\frac{\omega^{\prime}}{\theta_{\phi}^{\prime} \sin ^{2} \theta_{z}^{\prime}}=\frac{\lambda c}{4 n_{c o}^{2} \rho^{2}} m \quad \text { (maxima) } \\
& \frac{\omega}{\theta_{\phi} \sin ^{2} \theta_{z}}-\frac{\omega^{\prime}}{\theta_{\phi}^{\prime} \sin ^{2} \theta_{z}^{\prime}}=\frac{\lambda c}{8 n_{c o}^{2} \rho^{2}}(2 m+1) \quad \text { (minima) }
\end{aligned}
$$

In a more intuitive way, introducing the expression for the invariant $\bar{\beta}=n_{c o} \cos \theta_{z}$ [6] of each ray in the above expressions and reordering terms, we would get the above relations in the form:

$$
\begin{aligned}
& \frac{\omega}{\theta_{\phi}\left(n_{c o}^{2}-\beta^{2}\right)}-\frac{\omega^{\prime}}{\theta_{\phi}^{\prime}\left(n_{c o}^{2}-\beta^{2}\right)}=\frac{\lambda c}{4 n_{c o}^{4} \rho^{2}} m \quad \text { (maxima) } \\
& \frac{\omega}{\theta_{\phi}\left(n_{c o}^{2}-\beta^{2}\right)}-\frac{\omega}{\theta_{\phi}^{\prime}\left(n_{c o}^{2}-\beta^{\prime 2}\right)}=\frac{\lambda c}{8 n_{c o}^{4} \rho^{2}}(2 m+1) \quad \text { (minima) }
\end{aligned}
$$

From these completely general results, we can particularize three cases of great practical importance:

\subsection{Rays with Similar Incidence Angles}

In this case,

$$
\begin{aligned}
& \frac{\omega}{\theta_{\phi}}-\frac{\omega^{\prime}}{\theta_{\phi}^{\prime}}=\frac{\lambda c \sin ^{2} \theta_{z}}{4 n_{c o}^{2} \rho^{2}} m \quad \text { (maxima) } \\
& \frac{\omega}{\theta_{\phi}}-\frac{\omega^{\prime}}{\theta_{\phi}^{\prime}}=\frac{\lambda c \sin ^{2} \theta_{z}}{8 n_{c o}^{2} \rho^{2}}(2 m+1) \quad \text { (minima) }
\end{aligned}
$$

As a function of the invariant $\beta$, this condition is equivalent to $\beta^{2} \approx \beta^{\prime 2}$ in which case Eqs. (11a) and (11b) 
would take the form:

$$
\begin{aligned}
& \frac{\omega}{\theta_{\phi}}-\frac{\omega^{\prime}}{\theta_{\phi}^{\prime}}=\frac{\lambda c}{4 n_{c o}^{4} \rho^{2}}\left(n_{c o}^{2}-\beta^{2}\right) m \quad \text { (maxima) } \\
& \frac{\omega}{\theta_{\phi}}-\frac{\omega^{\prime}}{\theta_{\phi}^{\prime}}=\frac{\lambda c}{8 n_{c o}^{4} \rho^{2}}\left(n_{c o}^{2}-\beta^{2}\right)(2 m+1) \quad \text { (minima) }
\end{aligned}
$$

\subsection{Particular Case of One of the Rays Parallel to the Axis of the Fiber}

Let us consider the case in which one of the rays, for example $S^{\prime}$, is parallel to the axis of the fiber, that is, $\theta_{z}^{\prime}=0$ and $\theta_{\phi}^{\prime}=\frac{\pi}{2}$. In this case, equation (3) takes the form:

$$
\Delta_{S, S^{\prime}}=\bar{P} \bar{Q} n_{c o}\left(\frac{1}{\cos \theta_{z} \sin \theta_{\phi}}-1\right)
$$

and for the phase difference

$$
\delta_{S, S^{\prime}}=\frac{4 \pi}{\lambda}\left(\frac{\omega \rho^{2} n_{c o}^{2}}{c \theta_{\phi} \sin ^{2} \theta_{z}}-n_{c o} \bar{P} \bar{Q}\right)
$$

Applying conditions (8a) and (8b) for interference maxima and minima and making the new hypothesis $n_{c o}^{2}-\beta^{2} \approx n_{c o}^{2}$, we get:

$$
\begin{aligned}
& \frac{2 \omega \rho^{2} n_{c o}^{2}}{c \theta_{\phi}}-n_{c o} \bar{P} \bar{Q}=m \lambda \quad \text { (maxima) } \\
& \frac{4 \omega \rho^{2} n_{c o}^{2}}{c \theta_{\phi}^{2}}-n_{c o} \bar{P} \bar{Q}=(2 m+1) \lambda \quad(\text { minima })
\end{aligned}
$$

This result for the first time gives us an effective and simple tool to determine in the laboratory the rotation velocity $\omega$ associated with a given ray: it would suffice to adjust the length $\overline{P Q}$ to achieve stable interference patterns.

\subsubsection{Radiation with a very Short Wavelength}

The above results can be simplified enormously in one case that is very general -that is, that of electromagnetic radiation with a wavelength that is negligible compared with the dimensions of the fiber considered.

In this case, $n_{c 0} \overline{P Q} \gg m \lambda$ and therefore, we can get $n_{\infty} \overline{P Q} \gg m \lambda \approx n_{c 0} \overline{P Q}$. This means that the expressions (14a) y (14b) finally take the form:

$$
\begin{aligned}
& \omega=\frac{c \theta_{\phi} \bar{P} \bar{Q}}{2 \rho^{2} n_{c o}} \quad \text { (maxima) } \\
& \omega=\frac{c \theta_{\phi} \bar{P} \bar{Q}}{4 \rho^{2} n_{c o}} \quad \text { (minima) }
\end{aligned}
$$

Or, if the ray that is not parallel is meridional,

$$
\begin{aligned}
& \omega=\frac{c \pi \bar{P} \bar{Q}}{4 \rho^{2} n_{c o}} \quad \text { (maxima) } \\
& \omega=\frac{c \pi \bar{P} \bar{Q}}{8 \rho^{2} n_{c o}} \quad \text { (minima) }
\end{aligned}
$$

From the above expressions, we can deduce that, for short wavelengths, the rotation velocity of the guide when one of the rays is parallel to the axis does not depend on the order of the flank but on the optical path followed, i.e. the point at which the interference pattern is projected.

\section{CONCLUSIONS}

The above expressions, both the most general ones (10a)(10b) as well as the most particular ones (12a)-(12b), (14a)(14b) and (16a)-(16b) indicate that to give rise to an interference pattern, the modes propagated within an optical fiber must fulfill certain conditions with respect to each other.

It should be noted that for the modes to be propagated through the interior of an optical fiber, the fiber imposes certain restrictions on the propagation of these modes. However, so that they can also interfere with each other under classical interference conditions (coherence, monochromaticity, etc.), we add a restriction on the relative parameters of both modes with respect to each other.

Within the rotating-waveguide model, these restrictions are translated into values determined from the difference between the angular velocities $\omega \mathrm{y} \omega^{\prime}$ for each interference flank, now corresponding to a maximum or a minimum.

In the framework of the model, the meaning of the expressions is clear: each interference flank behaves as the sum (or difference) of two different modes, each of which has its characteristic parameters within the model. That is, given that the interference phenomenon consists of energy redistribution and that the rotating-waveguide model associates each mode with a rotation velocity, the result of applying this model to the phenomenon of the interferences is the splitting of each ray into various rotation velocities to interfere later with the velocities of the other and thus create an interference flank.

This result, completely original, is not deduced from the classical propagation model of the radiant energy within optical fibers [7, 8] and constitutes evidence of the clear correspondence between the rotation velocities of the hypothetical waveguides and the modes propagated within each fiber at the same time as it combines the geometric and electromagnetic descriptions of the propagation of radiation within optical fibers. 


\section{ACKNOWLEDGMENT}

We thank David Nesbitt for translating the text into English.

\section{REFERENCES}

1. F. Pérez-Ocón, A. Peña, J. R. Jiménez, and J. A. Díaz, “A simple model for fibre optics: planar dielectric waveguides in rotation," Eur. J. Phys. 27, 657-665 (2006).

2. A. P. García, F. Pérez-Ocón, and J. R. Jiménez, "Planar dielectric waveguides in rotation are optical fibers: comparison with the classical model," Opt. Express 16, 927-936 (2008).

3. A. P. García, F. Pérez-Ocón, and J. R. Jiménez, "The rotating planar dielectric waveguide model in wave optics: results for step-index profile optical fibers,” J. Opt. 12, 035103 (2010).

4. P. Zhang, S. Huang, Y. Hu, D. Hernández, and Z. Chen, "Generation and nonlinear self-trapping of optical propelling beams," Opt. Lett. 35, 3129-3131 (2010).

5. M. Born and E. Wolf, Principles of Optics: Electromagnetic Theory of Propagation, Interference and Diffraction of Light (Pergamon Press, New York, USA, 1999).

6. B. E. A. Saleh and M. C. Teich, Fundamentals of Photonics (Wiley Series in Applied Optics, New York, USA, 1991).

7. A. W. Snyder and J. D. Love, Optical Waveguide Theory (Chapman and Hall, New York, USA, 1983).

8. P. K. Cheo, Fiber Optics and Optoelectronics (Prentice Hall, NJ, USA, 1990). 